\title{
Factores de riesgo asociados al consumo problemático de alcohol en la adolescencia: El rol preventivo de mindfulness
}

\author{
José Manuel Lerma-Cabrera ${ }^{1}$, Philipp Steinebach ${ }^{2}$, Francisca Carvajal ${ }^{1}$, Valentina Ulloa ${ }^{3}$, \\ Cristian Cid-Parra ${ }^{4}$ y Álvaro I. Langer** \\ ${ }^{1}$ Universidad Autónoma de Chile, Santiago, Chile, ${ }^{2}$ University of Heidelberg, \\ ${ }^{3}$ University of Manchester, ${ }^{4}$ Pontificia Universidad Católica de Chile
}

(Recibido 4 Febrero, 2015; Aceptado 21 Febrero, 2015)

RESUMEN: El consumo problemático de alcohol en jóvenes se ha asociado con graves consecuencias sociales, de salud y económicas, generando enormes costos para los sistemas de la salud. Diversos estudios muestran que cuanto más temprano comienza el uso de sustancias, mayor es la probabilidad de desarrollar un trastorno de consumo de sustancias en la adultez. Sin embargo, esta relación se encuentra mediada por diversos factores de riesgo tanto contextuales (p.ej., historia de alcoholismo familiar) como individuales (p.ej., descontrol de impulsos). Los programas de prevención van dirigidos fundamentalmente a tratar de disminuir el consumo problemático y los daños de un consumo existente. Entre las aproximaciones psicológicas que mayor desarrollo han tenido en los últimos años se encuentran las intervenciones basadas en mindfulness (MBI) o también denominadas terapias-cognitivo conductuales de tercera generación. Recientemente las MBI se han utilizado en el tratamiento de la dependencia de sustancias. No obstante, son escasos los estudios que analizan el rol que mindfulness puede desempeñar a nivel de prevención sobre los factores asociados a un consumo de alcohol de riesgo y de los problemas asociados con este consumo en adolescentes.

Palabras clave: mindfulness, consumo de alcohol, adolescencia, prevención, salud mental.

Risk factors associated with problematic alcohol use in adolescence: The preventive role of mindfulness

ABSTRACT: Problematic alcohol consumption in youth is known to be related to social, health and financial problems, generating huge costs for the public health system. A number of studies describe early onset of drinking as being associated with an increased risk for developing alcohol dependence. However, this relationship is mediated by contextual (e.g. family history of alcoholism) and individual (e.g. problems of impulse control) risk factors. Therefore, prevention programs aim to reduce both problematic drinking and detriments of existing consumption. One of the most developed psychological approaches in recent years is mindfulness-based interventions (MBIs), also termed a part of third wave cognitive behavioral therapies. Recently, MBIs were applied in the treatment of substance dependence. Nevertheless, there are only a few studies 
which intend to analyze the role of mindfulness in the field of prevention, examining its effects on associated factors of risky alcohol use and related problems of this consumption in youth.

Keywords: mindfulness, alcohol consumption, adolescence, prevention, mental health.

\section{INTRODUCCIÓN}

Según la Organización Panamericana de Salud (2010), la edad de inicio de consumo de drogas ilícitas se encuentra en los 13 años, siendo la tendencia de algunos países intervenir desde la prevención para disminuir el ingreso al consumo (Maddaleno y Morello, 2003). A nivel mundial, se puede observar que un 2,8 $\%$ de la mortalidad es causada por el consumo de alcohol y que este porcentaje está creciendo (Rehm y Shields, 2013). Además, Shield, Parry y Rehm (2013) indican que el impacto del consumo de alcohol en trastornos y enfermedades crónicas conlleva costos enormes para los sistemas de la salud.

De hecho, cuanto más temprano comienza el uso de sustancias adictivas, mayor es la probabilidad de desarrollar un trastorno de consumo de estas sustancias en la adultez (Dennis, Muck, Dawud-Noursi y McDermeit, 2003). Sin embargo, estudios longitudinales desarrollados durante trece años, han mostrado que la relación entre el inicio temprano en el consumo de alcohol y el mayor riesgo a sufrir problemas asociados con el alcohol en la adultez está mediada por la presencia de diversos factores de riesgo (Norström y Pape, 2012; Rossow y Kuntsche, 2013).

El consumo problemático de alcohol en jóvenes se ha asociado con graves consecuencias sociales, de salud y económicas. Claramente, este tipo de consumo y sus consecuencias son un gran problema de salud pública al que la sociedad debe hacer frente. Así, los programas de prevención van dirigidos fundamentalmente a tratar de disminuir el consumo problemático y los daños de un consumo existente (Espada, Gonzálvez, Orgilés, Lloret y Guillén-Riquelme, 2015).

Entre las aproximaciones psicológicas que mayor desarrollo han tenido en los últimos años se encuentran las terapias cognitivo conductuales de tercera generación (Zgierska et al., 2009). Entre estas intervenciones se encuentran las Intervenciones basadas en Mindfulness (MBIs), la Terapia Dialéctica Conductual (DBT) o la Terapia de Aceptación y Compromiso (ACT). Los principales exponentes de las MBIs, son la Terapia Cognitiva basada en Mindfulness (MBCT) o la Terapia de Reducción de Estrés basada en Mindfulness (MBSR). Estas intervenciones han demostrado su efectividad en una amplia gama de problemas de salud mental, particularmente en depresión y ansiedad (Hofmann, Sawyer, Witty y Oh, 2010).

Recientemente las MBIs se han utilizado en el tratamiento de la dependencia de sustancias (Zgierska et al., 2009) y en intervenciones destinadas a la prevención de un consumo problemático en adolescentes. Sin embargo, son escasos los estudios que analizan el rol que mindfulness puede desempeñar a nivel de prevención sobre los factores asociados a un consumo de alcohol de riesgo y de 
los problemas asociados con este consumo en adolescentes.

\section{Intervenciones basadas en Mindfulness}

Mindfulness se ha descrito como "prestar atención de un modo particular: con un propósito, en el momento presente y sin establecer juicios de valor" (Kabat-Zinn, 1994, p. 15). Ha sido conceptualizado como un constructo teórico (mindfulness o conciencia plena), como una práctica (la meditación mindfulness) o como un proceso psicológico (ser o estar en mindfulness) (Germer, Siegel y Fulton, 2005).

El entrenamiento en mindfulness desde una aproximación occidental y académica se ha desarrollado y difundido a través de programas grupales estandarizados de ocho a diez semanas de duración ligados a contextos clínicos y de la salud, como MBSR y MBCT (Kabat-Zinn, 1990; Segal, Williams y Teasdale, 2002).

El objetivo del mindfulness es mantener la conciencia momento a momento, tomando distancia de las creencias, pensamientos o emociones, y por tanto, desarrollando un mejor balance emocional y bienestar (Ludwig y Kabat-Zinn, 2008). Para lograr esta experiencia, existen ciertos principios esenciales tales como: centrarse en el momento presente; la apertura a la experiencia y los hechos; laaceptación radical del momento actual; elección de las experiencias, y renuncia al control directo de emociones y pensamientos (Vallejo, 2006).

En la población adolescente, se han llevado a cabo una serie de estudios con muestras no clínicas, mayoritariamente en colegios, dando cuenta que los programas de mindfulness son efectivos en la disminución de síntomas ansiosos y depresivos, de la presión arterial, y del afecto negativo (Barnes, Davis, Murzynoeski y Treiber, 2004; Broderick y Metz, 2009; Lau y Hue, 2011; Raes, Griffith, Van DenGucht y Williams, 2014), así como en la mejora del autoconcepto, el rendimiento académico, la autoestima, la regulación de emociones, el bienestar psicológico, la fluidez y originalidad verbal (de la Fuente, Franco y Salvador 2010; Franco, 2009; Franco, Mañas, Cangas y Gallego, 2010; Langer, Ulloa, Cangas, Rojas y Krause, en prensa).

En universitarios también se han observado disminuciones en las puntuaciones en alexitimia y en dificultades en las habilidades sociales después de una intervención mindfulness (De la Fuente, Franco y Salvador, 2010), mientras que Langer, Cangas y Gallego (2010) concluyeron que una intervención basada en MBCT disminuía la ansiedad asociada a experiencias perceptuales estresantes y en la disminución de sintomatología depresiva (Gallego, Aguilar-Parra, Cangas, Langer y Mañas, 2014).

Por su parte, se ha visto que mindfulness también tiene efectos positivos en muestras adolescentes con variados problemas conductuales, emocionales o del desarrollo. Por ejemplo, una intervención de MBSR en pacientes adolescentes con patologías heterogéneas llevada a cabo en un centro psiquiátrico ambulatorio mostró provocar una disminución en síntomas de ansiedad, depresión y estrés 
somático, y un aumento en la autoestima y calidad del sueño (Biegel, Brown, Shapiro y Schubert, 2009).

También se ha probado su eficacia en la disminución de los niveles de agresividad en una muestra de estudiantes en riesgo de exclusión social y de adolescentes con Asperger (Singh et al., 2007; Singh et al., 2011). Bögels, Hoogstad, Dun, Schutter y Restifo (2008) encontraron, por otro lado, que la meditación mindfulness ayudaba a mejorar las metas personales, las preocupaciones internas y externas, los problemas de atención, el nivel de felicidad y la conciencia mindfulness en adolescentes con trastornos externalizantes como el déficit atencional, oposicionista-desafiante y del espectro autista.

Así, mindfulness puede ser un enfoque apropiado para lidiar con los problemas que enfrentan los adolescentes, demostrando tener una base empírica que apoya su viabilidad y aceptabilidad en esta población (Burke, 2009). En este sentido, podría desempeñar un rol relevante en la prevención de los factores de riesgos asociados al consumo problemático en adolescentes y jóvenes.

\section{Factores de riesgo de un consumo de alcohol problemático}

\section{Contextuales y Sociales}

Diversos estudios se han centrado en conocer cuáles son los factores que juegan un papel fundamental en la predisposición de un sujeto a desarrollar abuso en el consumo de alcohol, basándose en la premisa de que si conocemos los factores que incrementan el riesgo de sufrir problemas con el alcohol en adolescentes es posible prevenir o atenuar su consumo.

Uno de los factores contextuales asociados con el uso de alcohol en la adolescencia es tener una historia de alcoholismo familiar, la cual se asocia con un mayor consumo de alcohol (Labrie, Migliori, Kenney y Lac, 2010) y con mayor frecuencia de problemas con el alcohol (Leeman, Fenton y Volpicelli, 2007). Otros factores contextuales que tienen un efecto sobre el inicio y la intensidad del consumo de alcohol son los eventos estresantes (Enoch, 2011) o el uso de drogas por parte de los amigos (Silveri, 2012).

En un estudio longitudinal desarrollado durante 16 años, Huurre et al. (2010) analizaron cómo los factores de riesgo, ocurridos durante la etapa juvenil, influyen en un consumo excesivo de alcohol en la adultez. Los factores más importantes en varones son: a) divorcio de los padres b) síntomas depresivos c) tiempo pasado con amigos y d) hábito de un consumo alto de alcohol. En mujeres jóvenes, los factores son: a) hábito de un consumo alto de alcohol y b) fumar frecuentemente.

Por su lado, Jones, Feinberg, Cleveland y Cooper (2012) analizaron datos psicosociales longitudinales durante 2 años de más de 100.000 estudiantes. Los resultados indicaron que asociaciones antisociales en los amigos, riesgos familiares, actitud y comportamiento antisocial individual así como factores protectores de la comunidad son los factores más importantes para la predicción del 
consumo de alcohol dos años después. Otro estudio mostró que la probabilidad de tener problemas con el alcohol en la adultez se relacionaba con una mayor frecuencia de consumo de alcohol durante la adolescencia y con estar expuestos a ambientes familiares y recreacionales donde el alcohol estaba presente (Toumbourou et al., 2014).

\section{Factores Individuales}

Numerosos estudios han demostrado que además de los factores contextuales y sociales asociados con el consumo de alcohol (y problemas asociados) en adolescentes, podemos encontrarnos con factores individuales. Algunos de los factores personales más estudiados en relación a este tema han sido un pobre control inhibitorio (Nixon, 2013; Leeman et al., 2014) o determinados rasgos de personalidad como búsqueda de sensaciones (Noel, 2014) o impulsividad (Pearson y Henson, 2013). Todos estos factores muestran una correlación positiva con el uso de drogas en los jóvenes.

Precisamente, Pearson y Henson (2013) observaron que los adolescentes con una mayor impulsividad y falta de control inhibitorio, muestran mayores problemas relacionados con el alcohol. Estos autores destacan la importancia de considerar el control inhibitorio y la impulsividad para evaluar los problemas asociados con el consumo de alcohol.

Sin embargo, hay que señalar que el pobre control inhibitorio puede ser tanto la causa como la consecuencia de un consumo excesivo de alcohol. Así, indudablemente un pobre control inhibitorio puede predisponer a una persona a consumir alcohol, aumentando en consecuencia el riesgo de un consumo problemático. Pero por otro lado, también se sabe que el alcohol puede a su vez alterar el control inhibitorio o la impulsividad, comprometiendo por tanto la habilidad del sujeto para detener el consumo (López-Caneda, Rodríguez Holguin, Cadaveira, Corral y Doallo, 2014).

Por otra parte, una de las variables personales que más se ha asociado al consumo de alcohol es la presencia de problemas emocionales, los cuales pueden tanto gatillar como mantener el consumo (Rojas et al., 2012). Sin embargo, recientes estudios longitudinales muestran que hay diferencias de género en esta asociación. Por ejemplo, Saraceno, Heron, Munafò, Craddock y van den Bree (2012) demostraron que solo en mujeres, presentar síntomas depresivos a los 10 años, se asocia tres años más tarde, con un aumento en el riesgo de presentar un consumo problemático de alcohol en la adolescencia temprana.

\section{Mindfulness y su rol protector del consumo problemático}

Aunque la gran mayoría de los estudios que han evaluado la aplicabilidad y eficacia de MBI en problemas de dependencia al alcohol se han enfocado en población adulta (p.ej. Bowen et al., 2009; Brewer, Bowen, Smith, Marlatt y Potenza, 2010; Zgierska et al., 2009), existe evidencia de la aplicación de mindfulness en adolescentes con problemas de consumo excesivo de alcohol. 
Por ejemplo, un grupo de investigadores aplicó una intervención multicomponente, que incluye MBSR, para mejorar el sueño en adolescentes que habían recibido tratamiento por abuso de sustancias. Sus resultados arrojaron evidencia de mejoras en la calidad del sueño, el estrés emocional, y reducción en el consumo de sustancias (Bootzin y Stevens, 2005; Britton et al., 2010; Cousins, Bootzin, Stevens, Ruiz y Haynes, 2007). Además, Britton y colaboradores (2010) encontraron que los adolescentes a las 60 semanas de seguimiento mantenían la reducción del consumo de sustancias y del estrés emocional.

Además de evaluar las intervenciones, hay estudios que averiguan la relación entre mindfulness como proceso psicológico y el consumo de sustancias. Karyadi, VanderVeen y Cyders (2014) muestraron una relación baja pero significativa entre mindfulness y consumo de diferentes sustancias en un meta-análisis. Bramm, Cohn y Hagmann (2013) demostraron que mindfulness está asociado negativamente con consumo problemático de alcohol. De esa manera, los autores describen mindfulness como un factor protector de problemas asociados con el consumo de alcohol en estudiantes. Además, Fernandez, Wood, Stein y Rossi (2010) encontraron una correlación negativa baja pero significativa entre aspectos de mindfulness y consecuencias problemáticas del consumo de alcohol.

Analizando posibles factores que influyen en la relación entre mindfulness y consumo problemático de alcohol, Bodenlos y Noonan (2013) reportaron el estrés percibido como mediador en estudiantes. Así, se puede suponer que personas con niveles altos de mindfulness perciben menos estrés psicológico y por lo tanto, una tendencia reducida hacia el consumo problemático de alcohol. Respecto al nivel del consumo de alcohol, el estudio de Adams et al. (2015) mostró resultados que describen mindfulness como moderador de la relación entre estrés psicológico y consumo de alcohol.

Aparte del estrés psicológico, el impacto de mindfulness en la impulsividad puede influir en el consumo problemático de alcohol. Así, Murphy y MacKillop (2012) describieron en un estudio con adultos jóvenes que la correlación negativa entre mindfulness y abuso de alcohol se basa en diferentes niveles de impulsividad. Consecuentemente, personas con altos niveles de mindfulness presentarían niveles más bajos de impulsividad. En un estudio sobre control ejecutivo, mindfulness y percepción de alcohol, Ostafin, Kassman y Wessel (2013) confirmron esta idea.

Tanto mindfulness como control ejecutivo, es decir, la habilidad de controlar la atención cognitiva para mantener o repasar información, están asociados negativamente con medidas implícitas de la valencia de alcohol y con preocupación con pensamientos de alcohol. De esta manera, un alto nivel de mindfulness y control ejecutivo podrían mejorar el autocontrol consciente mediante la separación entre asociaciones automáticas y respuestas consecutivas. En un estudio de intervención de mindfulness en estudiantes, Ostafin, Bauer y Myxter (2012), reportaron resultados que indican una separación de procesos mentales automáticos y consumo de alcohol mediante la técnica de mindfulness.

En estudios recientes, se puede ver que mindfulness puede mejorar el fun- 
cionamiento cognitivo. Especialmente las funciones ejecutivas -un conjunto de habilidades cognitivas que incluye planeamiento y ejecución de accionesmuestran asociaciones con mindfulness y procesos importantes en el consumo de sustancias en jóvenes. Flook et al. (2010) demostraron que intervenciones de mindfulness en niños pueden mejorar las funciones ejecutivas, especialmente en aquellos con niveles bajos de estas funciones. Las funciones ejecutivas representan habilidades cognitivas importantes para afrontar procesos automáticos. De esta manera, el mejoramiento de estas habilidades podría facilitar el autocontrol en jóvenes y evitar un consumo problemático.

\section{A modo de Integración}

Como se describió anteriormente, los procesos del consumo de alcohol en adolescentes son complejos y los efectos dependen de varios factores interrelacionados. Por eso, la inclusión de otras técnicas de intervención a nivel de prevención es un elemento crucial para disminuir efectos problemáticos del consumo de alcohol en este grupo etario. Dado que el ámbito escolar facilita intervenciones generales de prevención, mindfulness representa una técnica adecuada para niños y adolescentes en este contexto (Langer et al., en prensa; Zenner et al., 2014).

Entre los factores que median el consumo de alcohol entre adolescentes y jóvenes se encuentra el estrés percibido, el cual aumenta significativamente en periodos de exámenes (Álvarez-Hernández, Aguilar-Parra, Fernández-Campoy, Salguero-García y Pérez-Gallardo, 2013). Por otro lado, existe una importante asociación entre la presencia de síntomas depresivos y el consumo de alcohol en adolescentes (McCarty et al., 2012).

En este sentido, varios estudios demuestran efectos beneficiosos de la práctica mindfulness con respecto a la reducción de estrés, síntomas de ansiedad y depresión. De esta forma, mindfulness representaría un mediador entre estrés psicológico y consumo problemático de alcohol (Adams et al. 2013; Bodenlos y Noonan, 2013).

Los resultados descritos indican que mindfulness probablemente está más fuertemente asociado (inversamente) con el consumo problemático de alcohol, en vez de con el nivel del consumo general. Aparentemente, la reducción del estrés psicológico puede explicar parcialmente los efectos protectores de mindfulness.

Por otro lado, el entrenamiento en mindfulness, potencia el cambio en la atención hacia el momento presente y la aceptación de los pensamientos y sensaciones, lo que puede ayudar a los adolescentes a ganar un punto de vista no apegado y a aumentar la tolerancia a situaciones, a sentimientos displacenteros y al estrés que puede llevar al consumo de sustancias (Brewer et al., 2010).

En otras palabras, practicar mindfulness significa potenciar funciones cognitivas, especialmente la inhibición y control de impulsos, factores ampliamente relacionados con el consumo problemático de alcohol en adolescentes (Murphy 
y MacKillop, 2012; Ostafin, et al., 2013). En este sentido, los programas preventivos deberían considerar los procesos bidireccionales involucrados en el consumo problemático del alcohol como la interrelación entre los efectos del alcohol y los procesos cognitivos afectados por el consumo.

Los hallazgos científicos descritos en el presente artículo son sólo el comienzo de una relación que puede resultar extraordinariamente fructífera: la posibilidad de prevenir el consumo problemático de alcohol a través de la práctica de mindfulness. En otras palabras, proporcionar competencias socio-afectivas a los jóvenes y adolescentes podría desempeñar un rol protector frente al consumo problemático o de riesgo de alcohol. Específicamente, son recomendables estrategias de prevención que consideren el contexto natural de los jóvenes como son los establecimientos educativos (Langer et al., en prensa).

\section{AGRADECIMIENTOS}

Parte de este estudio contó con el apoyo de CONICYT/PAI, Concurso Nacional Apoyo al Retorno de Investigadores Desde el Extranjero, No 82130055 y del Fondo de Innovación para la Competitividad (FIC) del Ministerio de Economía, Fomento y Turismo, a través de la Iniciativa Científica Milenio, Proyecto IS130005.

\section{REFERENCIAS}

Álvarez-Hernández, J., Aguilar-Parra, J.M., Fernández-Campoy, J.M., SalgueroGarcía. D. y Pérez Gallardo, E.R. (2013). El estrés ante los exámenes en los estudiantes universitarios. Propuesta de intervención. International Journal of Developmental and Educational Psychology, 1, 179-188.

Adams, C. E., Cano, M. A., Heppner, W. L., Stewart, D. W., Correa-Fernández, V., Vidrine, J. I. (2015). Testing a moderated mediation model of mindfulness, psychosocial stress, and alcohol use among African American smokers. Mindfulness.

Barnes, V. A., Davis, H. C., Murzynowski, J. B. y Treiber, F. A. (2004). Impact of meditation on resting and ambulatory blood pressure and heart rate in youth. Psychosomatic Medicine, 66 (6). Retrieved from http://journals.lww.com/ psychosomaticmedicine/Fulltext/2004/11000/Impact_of_Meditation_on_ Resting_and_Ambulatory.17.aspx

Biegel, G., Brown, K., Shapiro, S. y Schubert, C. (2009). Mindfulness-based stress reduction for the treatment of adolescent psychiatric outpatients: A randomized clinical trial. Journal of Consulting and Clinical Psychology, 77, $855-866$.

Bodenlos, J. S., Noonan, M. y Wells, S. Y. (2013). Mindfulness and alcohol problems in college students: The mediating effects of stress. Journal of American College Health, 61, 371-378.

Bögels, S., Hoogstad, B., Dun, L., Schutter, S. y Restifo, K. (2008). Mindfulness 
training for adolescents with externalizing disorders and their parents. Behavioral and Cognitive Psychotherapy, 36, 193- 209.

Bootzin, R. R. y Stevens, S. J. (2005). Adolescents, substance abuse, and the treatment of insomnia and daytime sleepiness. Clinical Psychology Review, 25, 629-644.

Bowen, S., Chawla, N., Collins, S., Witkiewitz, K., Hsu, S., Grow, J., et al. (2009). Mindfulness-based relapse prevention for substance disorders: A pilot efficacy trial. Substance Abuse, 30, 295-305.

Bramm, S. M., Cohn, A. M. y Hagman, B. T. (2013). Can preoccupation with alcohol override the protective properties of mindful awareness on problematic drinking? Addictive Disorders and Their Treatment, 12, 19-27.

Brewer, J., Bowen, S., Smith, J., Marlatt, A. y Potenza, M. (2010). Mindfulnessbased treatments for co-occurring depression and substance use disorders: What can we learn from the brain? Addiction, 105, 1698-1706.

Britton, W., Bootzin, R., Cousin, J., Hasler, B., Peck, T. y Shapiro, S. (2010). The contribution of mindfulness practice to a multicomponent behavioral sleep intervention following substance abuse treatment in adolescents: A treatment-development study. Substance Abuse, 31, 86-97.

Broderick, P. C. y Metz, S. (2009). Learning to BREATHE: A Pilot Trial of a Mindfulness Curriculum for Adolescents. Advances in School Mental Health Promotion, 2, 35-46.

Burke, C. (2009). Mindfulness-based approaches with children and adolescents: A preliminary review of current research in an emergent field. Journal of Children Farmacological Studies, 19, 133-144.

Cousins, J. C., Bootzin, R. R., Stevens, S. J., Ruiz, B. S. y Haynes, P. L. (2007). Parental involvement, psychological distress, and sleep: A preliminary examination in sleep-disturbed adolescents with a history of substance abuse. Journal of Family Psychology, 21, 104-113.

De la Fuente, M., Franco, C. y Salvador, M. (2010). Efectos de un programa de meditación (mindfulness) en la medida de la alexitimia y las habilidades sociales. Psicothema, 22, 369-375.

Dennis, M. L., Muck, R. D., Dawud-Noursi, S. y McDermeit, M. (2003). The need for developing and evaluating adolescent treatment models. In S. J. Stevensy A. R. Morral (Eds.). Adolescent substance abuse treatment in the United States: Exemplary models from a National Evaluation Study (pp. 3-34). Binghampton, NY The Haworth Press.

Enoch, M.A. (2011). The role of early life stress as a predictor for alcohol and drug dependence. Psychopharmacology,214,17-31.

Espada, J.P., Gonzálvez, M.T., Orgilés M, Lloret, D. y Guillén-Riquelme, A. (2015). Meta-analysis of the effectiveness of school substance abuse prevention programs in Spain. Psicothema, 27, 5-12.

Fernandez, A. C., Wood, M. D., Stein, L. A. R. y Rossi, J. S. (2010). Measuring mindfulness and examining its relationship with alcohol use and negative consequences. Psychology of Addictive Behaviors, 24, 608-616. 
Flook, L., Smalley, S. L., Kitil, M. J., Galla, B. M., Kaiser-Greenland, S., Locke, J. (2010). Effects of mindful awareness practices on executive functions in elementary school children. Journal of Applied School Psychology, 26, 70-95.

Franco, C. (2009). Reducción de la percepción de estrés en estudiantes de Magisterio mediante la práctica de la meditación fluir. Apuntes de Psicología, 27, 99-109.

Franco, C., Mañas, I., Cangas, A. y Gallego, J. (2010). The Applications of Mindfulness with Students of Secondary School: Results on the Academic Performance, Self-concept and Anxiety. In M. Lytras, P. Ordonez De Pablos, A. Ziderman, A. Roulstone, H. Maurery J. Imber (Eds.), Communications in Computer and Information Science. Knowledge Management, Information Systems, E-Learning, and Sustainability Research (pp. 83-97). Springer Berlin Heidelberg.

Gallego, J. Aguilar-Parra, J.M., Cangas, A.J., Langer, A.I. y Mañas, I. (2014). Effect of a mindfulness program on stress, anxiety, and depression in university students. Spanish Journal of Psychology, 17 (e109), 1-6.

Germer, C., Siegel, R. y Fulton, P (2005). Mindfulness and psychotherapy. New York: Guilford Press.

Hofmann, S. G., Sawyer, A. T., Witt, A. A.y Oh, D. (2010). The effect of mindfulness-based therapy on anxiety and depression: A meta-analytic review. Journal of Consulting and Clinical Psychology, 78, 169-183.

Huurre, T., Lintonen, T., Kaprio, J., Pelkonen, M., Marttunen, M. y Aro, H. (2010). Adolescent risk factors for excessive alcohol use at age 32 years. A 16-year prospective follow-up study. Social Psychiatry and Psychiatric Epidemiology, 45, 125-134.

Jones, D. E., Feinberg, M. E., Cleveland, M. J. y Cooper, B. R. (2012). A multidomain approach to understanding risk for underage drinking: converging evidence from 5 data sets. American Journal of Public Health, 102, 20802087.

Kabat-Zinn, J. (1990). Full Catastrophe Living: Using the wisdom of your body and mind to face stress, pain and illness. New York: Hyperion.

Kabat-Zinn, J. (1994). Wherever you go, there you are: Mindfulness meditation in everyday life. New York: Hyperion.

Karyadi, K. A., VanderVeen, J. D. y Cyders, M. A. (2014). A meta-analysis of the relationship between trait mindfulness and substance use behaviors. Drug and Alcohol Dependence, 143, 1-10.

Kuyken, W., Weare, K., Ukoumunne, O. C., Vicary, R., Motton, N., Burnett, R. et al., (2013). Effectiveness of the Mindfulness in Schools Programme: nonrandomised controlled feasibility study. The British Journal of Psychiatry, 203, 126-131.

Labrie, J. W., Migliuri, S., Kenney, S.R. y Lac, A. (2010). Family history of alcohol abuse associated with problematic drinking among college students. Addictive Behaviors, 35, 721-725.

Langer, A.I., Ulloa, J.V., Cangas, A.J., Rojas, G. y Krause, M. (En prensa). In- 
tervenciones basadas en mindfulness en educación secundaria: Una revisión sistemática cualitativa. Estudios de Psicología.

Langer A.I., Cangas A. y Gallego J. (2010). Mindfulness-based intervention on distressing hallucination like experiences in a nonclinical sample. Behaviour Change, 27, 176-183.

Lau, N.-s. y Hue, M.-t. (2011). Preliminary outcomes of a mindfulness-based programme for Hong Kong adolescents in schools: Well-being, stress and depressive symptoms. International Journal of Children's Spirituality, 16, 315-330.

Leeman, R.F., Fenton, M y Volpicelli, J. R. (2007) Impaired control and undergraduate problem drinking. Alcohol and Alcoholism, 42, 42-48.

Leeman, R. F., Beseler, C. L., Helms, C. M., Patock-Peckham, J. A., Wakeling, V. A. y Kahler, C. W. (2014). A brief, critical review of research on impaired control over alcohol use and suggestions for future studies. Alcoholism: Clinical and Experimental Research, 38, 301-308.

López-Caneda, E., Rodriguez Holguin, S., Cadaveira, F., Corral, M.y Doallo, S. (2014). Impact of alcohol use on inhibitory control (and vice versa) during adolescence and young. Alcohol and Alcoholism, 49,173-181.

Ludwig, D. y Kabat-Zinn, J. (2008). Mindfulness in medicine. Journal of the American Medical Association, 300, 1350- 1352.

McCarty, C. A., Wymbs, B. T., King, K. M., Mason, W. A., Stoep, A. V., McCauley, E. y Baer, J. (2012). Developmental consistency in associations between depressive symptoms and alcohol use in early adolescence. Journal of Studies on Alcohol and Drugs, 73, 444-453.

Maddaleno, M. y Morello, P. (2003). Salud y desarrollo de adolescentes y jóvenes en Latinoamérica y El Caribe: Desafíos para la próxima década. Salud Pública de Mexico, 45, 132-139.

Metz, S. M., Frank, J. L., Reibel, D., Cantrell, T., Sanders, R. y Broderick, P. C. (2013). The effectiveness of the learning to breathe program on adolescent emotion regulation. Research in Human Development, 10, 252-272.

Murphy, C. y MacKillop, J. (2012). Living in the here and now: Interrelationships between impulsivity, mindfulness, and alcohol misuse. Psychopharmacology, 219, 527-536.

Nixon, S. J. (2013). Executive functioning among young people in relation to alcohol use. Current Opinion in Psychiatry, 26, 305-309.

Noel, X. (2014). Why adolescents are at risk of misusing alcohol and gambling. Alcohol and Alcoholism, 49, 165-172.

Norstrom, T. y Pape, H. (2012). Associations between adolescent heavy drinking and problem drinking in early adulthood: Implications for prevention. Journal of studies on alcohol and drugs, 73, 542-548.

Organización Panamericana de Salud. (2010). Estrategia y plan de acción regional sobre los adolescentes y jóvenes. Washington, D.C.: OPS.

Ostafin, B. D., Bauer, C. y Myxter, P. (2012). Mindfulness decouples the relation between automatic alcohol motivation and heavy drinking. Journal of Social 
and Clinical Psychology, 31, 729-745.

Ostafin, B. D., Kassman, K. T. y Wessel, I. (2013). Breaking the cycle of desire: Mindfulness and executive control weaken the relation between an implicit measure of alcohol valence and preoccupation with alcohol-related thoughts. Psychology of Addictive Behaviors, 27, 1153-1158.

Pearson, M. R. y Henson, J. M. (2013). Unplanned drinking and alcohol-related problems: A preliminary test of the model of unplanned drinking behavior. Psychology of Addictive Behaviors, 27, 584-595.

Raes, F., Griffith, J. W., van der Gucht, K. y Williams, J. M. G. (2014). Schoolbased prevention and reduction of depression in adolescents: A cluster-randomized controlled trial of a mindfulness group program. Mindfulness, 5, 477-486.

Rehm, J. y Shield, K. D. (2013). Alcohol and mortality global alcohol-attributable deaths: From cancer, liver cirrhosis, and injury in 2010. Alcohol Research: Current Reviews, 35, 250-260.

Rojas, G., Gaete, J., Guajardo, V., Martínez, V., Barroilhet, S., Meneses, J. y Araya, R. (2012). Consumo de sustancias y síntomas depresivos en escolares de liceos municipales de Santiago. Revista Médica de Chile, 140, 184-191.

Rossow, I. y Kuntsche, E. (2013). Early onset of drinking and risk of heavy drinking in young adulthood-a 13-year prospective study. Alcoholism: Clinical and Experimental Research, 37, E297.

Saraceno, L. Heron, J.Munafò, M.Craddock, N y van den Bree, M.B. M. (2012). The relationship between childhood depressive symptoms and problem alcohol use in early adolescence: Findings from a large longitudinal populationbased study. Addiction, 103, 567-577.

Segal Z., Williams J. y Teasdale, J. (2002). Mindfulness based cognitive therapy for depression. New York: Guilford.

Shield, K. D., Parry, C. y Rehm, J. (2013). Chronic diseases and conditions related to alcohol use. Alcohol Research: Current Reviews, 35, 155-171..

Silveri, M. M. (2012). Adolescent Brain Development and Underage Drinking in the United States: Identifying Risks of Alcohol Use in College Populations. Harvard Review of Psychiatry, 20, 189-200.

Singh, N. N., Lancioni, G. E., Singh, A. D. A., Winton, A. S. W., Singh, A. N. A. y Singh, J. (2011). Adolescents with Asperger syndrome can use a mindfulness-based strategy to control their aggressive behavior. Research in Autism Spectrum Disorders, 5, 1103-1109.

Singh, N. N., Lancioni, G. E., Winton, A. S. W., Singh, J., Curtis, W. J., Wahler, R. G. y McAleavey, K. M. (2007). Mindful parenting decreases aggression and increases social behavior in children with developmental disabilities. $\mathrm{Be}$ havior Modification, 31, 749-771.

Toumbourou, J. W., Evans-Whipp, T. J., Smith, R., Hemphill, S. A., Herrenkohl, T. I. y Catalano, R. F. (2014). Adolescent predictors and environmental correlates of young adult alcohol use problems. Addiction, 109, 417-424.

Vallejo, (2006). Mindfulness. Papeles del Psicólogo, 27, 92-99. 
Zenner, C., Herrnleben-Kurz, S. y Walach, H. (2014). Mindfulness-based interventions in schools a systematic review and meta-analysis. Frontiers in Psychology, 5, 603.

Zgierska, A., Rabago, D., Chawlas, N., Kushner, K., Koehler, R. y Marlatt, A. (2009). Mindfulness meditation for substance use disorders: A systematic review. Substance Abuse, 30, 266-294. 